\title{
Caffeine Use Disorder: A Review of the Evidence and Future Implications
}

\author{
Merideth A. Addicott
}

Published online: 28 May 2014

(C) Springer International Publishing AG 2014

\begin{abstract}
The latest edition of the Diagnostic and Statistical Manual of Mental Disorders (5th edition; DSM-5) has introduced new provisions for caffeine-related disorders. Caffeine withdrawal is now an officially recognized diagnosis, and criteria for caffeine use disorder have been proposed for additional study. Caffeine use disorder is intended to be characterized by cognitive, behavioral, and physiological symptoms indicative of caffeine use despite significant caffeinerelated problems, similar to other substance use disorders. However, since non-problematic caffeine use is so common and widespread, it may be difficult for some health professionals to accept that caffeine use can result in the same types of pathological behaviors caused by alcohol, cocaine, opiates, or other drugs of abuse. Yet there is evidence that some individuals are psychologically and physiologically dependent on caffeine, although the prevalence and severity of these problems is unknown. This article reviews the recent changes to the DSM, the concerns regarding these changes, and some potential impacts these changes could have on caffeine consumers.
\end{abstract}

Keywords Caffeine-related disorders · Diagnostic and Statistical Manual · DSM · Withdrawal · Dependence

\section{Introduction}

After centuries of cultivation and consumption, our relationship with caffeine has just undergone a major change. The latest version of the Diagnostic and Statistical Manual of

\footnotetext{
M. A. Addicott $(\square)$

Department of Psychiatry and Behavioral Sciences, Duke-UNC

Brain Imaging and Analysis Center, Duke University Medical

Center, 2608 Erwin Rd, Box 3527, Lakeview Pavilion E, suite 300,

Durham, NC 27705, USA

e-mail: merideth.addicott@duke.edu
}

Mental Disorders (5th edition; DSM-5) now includes caffeine withdrawal disorder and proposes a set of criteria for caffeine use disorder (CUD) [1]. What effect will this have on us and America's most popular psychostimulant?

Caffeine is generally considered a functional or beneficial drug because it can improve mood and alertness at low doses. At high doses, caffeine produces aversive intoxicating effects. For this reason, caffeine consumption is typically self-limiting and compatible with a social and productive life [2]. Caffeine is thought to have little to no abuse liability, but perhaps its modest reinforcing effects enhance the desirability of beverages that already have pleasant flavors and aromas, such as coffee, tea, and soft drinks. For many of us who sit behind computer screens all day, these caffeinated beverages help us focus our attention and provide a welcome excuse to get up from our chairs once in a while. Although the question of whether we are all collectively dependent on caffeine has been raised [3], coffee drinking is thought to be "more a dedicated habit than a compulsive addiction" [4].

The majority of people who use caffeine safely every day may find it difficult to understand how caffeine use could become disordered or problematic. Of course, many coffee drinkers probably have had a personal experience with withdrawal symptoms if they skipped their morning coffee, but the remedy for that is simply a cup of coffee. But what if someone were convinced he could not function without caffeine? What if he took increasingly greater amounts of caffeine to improve his ability to function, until he began to experience the effects of caffeine intoxication or withdrawal more days than not? What if he were told that his caffeine use was physically harming his body, but he could not reduce his use? At what point does caffeine use become disordered?

A few studies have suggested that some individuals meet the criteria for substance dependence regarding their caffeine use. However, many questions remain regarding the prevalence, development, and severity of disordered caffeine use. 
To help answer these questions and guide future research on this topic, the DSM-5 proposes a set of criteria for CUD. This article reviews the caffeine-related changes to the DSM and the recent research and evidence for disordered caffeine use.

\section{Diagnostic and Statistical Manual of Mental Disorders, 4th Edition (DSM-IV) Caffeine-Related Diagnoses}

\section{Caffeine Withdrawal}

The fourth edition of the DSM (DSM-IV) recognized four caffeine-related diagnoses: caffeine intoxication, caffeineinduced anxiety disorder, caffeine-induced sleep disorder, and caffeine-related disorder not otherwise specified (NOS) [5]. The criteria for caffeine intoxication included recent caffeine use, usually in excess of $250 \mathrm{mg}$, and five or more symptoms that develop shortly thereafter, such as restlessness, nervousness, insomnia, gastrointestinal disturbance, and tachycardia. Of the caffeine-related diagnoses included in the DSM-IV, caffeine withdrawal is notably absent, although a proposed set of criteria was included to encourage future research. The DSM-IV recognized that "some individuals who drink large amounts of coffee display some aspects of dependence on caffeine and exhibit tolerance and perhaps withdrawal. However, the data are insufficient at this time to determine whether these symptoms are associated with clinically significant impairment that meets the criteria for Substance Dependence or Substance Abuse" (page 212) [5].

Over the past 30 years, there have been a large number of studies characterizing caffeine withdrawal symptoms (for reviews, see Griffiths and Woodson [6] and Juliano and Griffiths [7]). As a result, the DSM-5 includes diagnostic criteria for caffeine withdrawal, which consists of prolonged daily use of caffeine and three or more withdrawal symptoms occurring within $24 \mathrm{~h}$ of abrupt cessation or reduction of caffeine use. These symptoms include headache, marked fatigue or drowsiness, dysphoric mood/depressed mood/irritability, difficulty concentrating, and flu-like symptoms [1].

\section{Caffeine Dependence}

Concurrent with research on caffeine withdrawal, investigators have also been studying caffeine's abuse potential. Although the DSM-IV included a substance dependence diagnosis for every other recognized substance, there were no criteria or proposed criteria for caffeine dependence. Therefore, investigators adapted the DSM-IV substance dependence criteria for caffeine use to use in their research. The criteria for substance dependence consisted of a maladaptive pattern of substance use with clinically significant impairment manifested by three or more symptoms within a 12-month period. These symptoms included (1) tolerance; (2) withdrawal; (3) substance used in larger amounts or over a longer period than intended; (4) a persistent desire or unsuccessful effort to control use; (5) a great deal of time spent obtaining, using, or recovering from the substance; (6) forgoing important activities because of the substance; and (7) substance use continued despite knowledge of having a persistent or recurrent physical or psychological problem likely to be caused or exacerbated by the substance (i.e., 'use despite harm') [5].

There have been four notable studies that have investigated caffeine dependence. Strain et al. recruited participants who believed they were psychologically or physiologically dependent on caffeine. The authors reported that 16 out of 27 subjects met three of four criteria for caffeine dependence, including tolerance, withdrawal, persistent desire/ unsuccessful efforts to control use, and 'use despite harm' (e.g., using caffeine against medical advice) [8]. A similar study by Juliano et al. reported that $93 \%$ of 94 subjects met three of seven criteria for caffeine dependence, and $55 \%$ of subjects met five of seven criteria. Most of the interviewees reported at least one serious attempt to quit or reduce caffeine without success and $43 \%$ were advised by a health professional to reduce caffeine use for health reasons (including cardiovascular problems, fibrocystic breast disease, pregnancy, anxiety, headaches, sleep difficulties, or to reduce caloric intake from caffeinated soft drinks) [9]. Another study by Striley et al. recruited subjects who were expected to have high rates of drug use/abuse. The authors reported that $35 \%$ of 167 subjects endorsed three of seven caffeine dependence criteria [10]. Lastly, Hughes et al. conducted random phone surveys of Vermont residents. They reported that $30 \%$ of 162 subjects endorsed three or more criteria, with the highest percentage of people endorsing a desire to control caffeine use, followed by spending a great deal of time with the drug, and using more caffeine than intended [11].

In summary, these studies suggest that caffeine use has the features of substance dependence for some individuals. Furthermore, they suggest that not all caffeine users can simply quit on their own, which is an attitude probably held by some health professionals [9]. However, these studies have several limitations. Three of the studies used targeted subject samples [8-10], and so the prevalence of caffeine dependence in the general population cannot be estimated. Additionally, in two of the studies, interviews were not conducted by psychiatric clinicians, so issues of severity and harm related to caffeine dependence may not have been adequately addressed $[10,11]$. The studies on caffeine dependence reviewed here were conducted prior to the publication of the DSM-5 in 2013. Although caffeine dependence did not become an officially recognized diagnosis in this edition, these and other studies 
elicited interest in the psychiatric community to learn more about disordered caffeine use.

\section{DSM, 5th Edition (DSM-5) Caffeine-Related Diagnoses}

The DSM-5 includes caffeine intoxication, caffeine withdrawal, other caffeine-induced disorders (e.g., anxiety and sleep disorders), and unspecified caffeine-related disorder. In this edition, substance abuse and substance dependence are now represented by substance use disorder (SUD), which is applied to all classes of substances except for caffeine. For this diagnosis, individuals must endorse at least two of the following criteria: (1) substance used in larger amounts or over longer period than intended; (2) a persistent desire or unsuccessful effort to control use; (3) a great deal of time spent obtaining, using, or recovering from the substance; (4) craving the substance; (5) substance use interfering with ability to fulfill major obligations; (6) substance use despite social problems related to use; (7) important occupational or social activities given up because of substance use; (8) recurrent use in situations when it is physically hazardous; (9) 'use despite harm'; (10) tolerance; and (11) withdrawal [1].

The DSM-5 does not include a diagnosis of CUD because, according to the American Psychiatric Association (APA), it is not yet clear to what extent it is a clinically significant disorder. However, CUD is included in Section III ("Emerging Measures and Models") of the DSM-5 to encourage further research on the impact of this condition [12]. The proposed CUD criteria are the same as other SUDs; however, the CUD diagnosis is designed to be more conservative. For a CUD diagnosis, all three of the following criteria need to be endorsed: (1) a persistent desire or unsuccessful effort to control use; (2) 'use despite harm'; and (3) withdrawal. This higher threshold is intended to prevent over-diagnosis of CUD given the prevalence of non-problematic caffeine use in the general population [1]. These proposed criteria are intended to encourage more research on the reliability, validity, and prevalence of CUD, as well as its functional consequences on the lives of those affected by it.

\section{Current Literature on Caffeine Use Disorder}

There are three notable articles that summarize the current attitudes and information regarding caffeine withdrawal and CUD. First, a roundtable discussion with Drs. Hughes, Griffiths, Juliano, and Budney provides an excellent overview of the caffeine-related changes to the DSM-5 and explains some of the decision-making process behind the revisions, as well as the concerns about caffeine withdrawal and CUD over-diagnosis [13••]. The discussants explain how the more conservative criteria for CUD than other SUDs should help prevent over-diagnosis, but, at the same time, diagnoses included in the DSM should not be exceedingly rare. Thus, more information is needed on the prevalence of CUD before deciding whether it belongs in the DSM. Another issue raised by the panel is that there is a common perception of caffeine being a functional drug; in fact, there has been a substantial amount of research on its benefits (for review, see Glade [14]). However, once caffeine (or any other substance) has been determined to be an addictive drug, then prejudices against discussing any potentially beneficial effects often develop in the psychiatric community $[13 \cdot \bullet]$. This conflict of interest could interfere with future caffeine research.

A second article complements some of the issues raised by the roundtable concerning attitudes among the psychiatric community, including both researchers and clinicians. Budney et al. (2013) investigated popular opinions about caffeine dependence/CUD among members of professional societies relevant to addiction. An overwhelming majority (95\%) of those surveyed believed that caffeine cessation can produce withdrawal and $73 \%$ thought withdrawal could have clinical importance, but fewer than half thought caffeine withdrawal should be in the DSM. A small majority (58 \%) of respondents thought that some individuals could develop CUD, and $44 \%$ believed CUD should be a DSM diagnosis [15]. These attitudes will be influenced by research published over the next few years and could affect what caffeine-related diagnoses are included in the next edition of the DSM.

Lastly, Meredith et al. provides a comprehensive review of studies on caffeine use/abuse/dependence and summarizes the existing evidence in support of the three primary CUD criteria. The authors also present a number of research directions needed to further support and understand CUD. As the authors note, the prevalence of CUD is difficult to estimate from existing studies since DSM-IV criteria for caffeine dependence were used previously and the current criteria for CUD are slightly different $[16 \bullet \bullet]$.

\section{Use Despite Harm}

Before CUD can become an official diagnosis, more research is needed on the severity of symptoms of the three primary criteria: (1) a persistent desire or unsuccessful effort to control use; (2) substance use continued despite knowledge of having a persistent or recurrent physical or psychological problem likely to be caused or exacerbated by the substance (i.e., 'use despite harm'); and (3) withdrawal [1]. In this author's opinion, criterion 2 is the most contentious issue and in need of clarification. Some authors appear to accept that caffeine consumption is associated with negative health effects (e.g., Juliano et al. [9] and Striley et al.[10]), while others believe that it is not (e.g., Morelli and Simola [2], Hughes et al. [11], 
and Lara [17]). These opinions can influence research directions and hypotheses; therefore, closer examination of this criterion is needed to promote consensus on what health problems can define ‘use despite harm' for CUD.

Evidence for Physical Problems Caused or Exacerbated by Caffeine Large, acute doses of caffeine are known to cause caffeine intoxication, which can cause a significant threat to one's health and require medical attention. McCarthy et al. reviewed caffeine-related calls to a state poison control center. Out of 254 reported cases of caffeine abuse, 106 patients were managed in an emergency department and 34 were hospitalized and/or admitted to an intensive care unit [18•]. In addition, Ogawa and Ueki presented two case reports of individuals whose daily caffeine use escalated until symptoms of caffeine intoxication made medical intervention necessary [19•]. Clearly, caffeine intoxication is a medically significant health problem. However, could an otherwise healthy individual meet the criterion for 'use despite harm' by consuming a low to moderate daily dose of caffeine? A review by Nawrot et al. on caffeine and health recommended that doses up to $400 \mathrm{mg} /$ day are safe [20]; however, it is difficult to determine the health effects of low to moderate daily doses of caffeine because the effects of caffeine cannot be easily separated from the effects of caffeinated beverages, usually coffee, tea, soft drinks, or energy drinks. The antioxidant effects of polyphenols in tea and coffee are thought to have health benefits, while the excess sugars in soft drinks and energy drinks can be detrimental. Despite these confounds, there has actually been a great deal of research on the health effects of caffeine. However, the data are inconsistent.

A review of the literature on caffeine and health is outside the scope of this article, but a brief example may be informative: caffeine causes a small, temporary increase in blood pressure in normotensive adults. Tolerance to these effects may develop in some people, but caffeine could pose a threat to patients with, or at risk for, hypertension. Some studies have suggested an increased risk of sustained hypertension following coffee consumption (e.g., Palatini et al. [21]), while others have not found a significant relationship (e.g., Klag et al. [22]). Even two recent meta-analyses on caffeine and hypertension arrived at different conclusions: one found no evidence of a relationship [23] and the other found an elevated risk of hypertension associated with 1-3 cups of coffee per day, but not with 3 or more cups per day [24].

Clinicians make recommendations to their patients based on their knowledge of the literature, but the literature on caffeine and health is enormous and complicated. In at least two of the studies on caffeine dependence, subjects met the criteria for 'use despite harm' if they admitted using caffeine against medical advice $[8,9]$. If a clinician who read about an association between caffeine and hypertension (e.g., Palatini et al. [21]) recommended to her hypertensive patient to stop drinking coffee and he did not, should that patient meet the criteria for 'use despite harm' even though another physician who read a different article (e.g., Klag et al. [22]) would not have made the same recommendation?

To this author's knowledge, low to moderate daily caffeine intake has not been proven to cause significant and irreversible health problems that would warrant medical intervention. That is not to say that caffeine does not or cannot have negative health effects, but researchers and clinicians need to agree on what physical health problems can be caused by chronic low to moderate caffeine intake. Whether or not low to moderate daily doses of caffeine can cause physical harm is an important issue to resolve since medical professionals recommend limiting/eliminating caffeine intake to some of their patients, and health concerns are a common reason for individuals to want to modify their caffeine use [9]. Furthermore, the fate of CUD in the next edition of the DSM may depend on the definition of 'use despite harm,' since the other two primary criteria for CUD (i.e., a persistent desire or unsuccessful effort to control use and withdrawal) could potentially be endorsed at any daily dose, even while consuming as little as $100 \mathrm{mg} /$ day [25].

Evidence for Psychological Problems Caused or Exacerbated by Caffeine The DSM-5 recognizes that some features of CUD may be positively associated with other psychiatric diagnoses [1], and there have been studies investigating whether caffeine use or withdrawal can exacerbate existing psychiatric symptoms. In particular, the anxiogenic effects of high caffeine doses can aggravate symptoms of anxiety, panic disorder, and insomnia (for reviews, see Lara [17] and Winston et al. [26]). In fact, a review of eight studies that administered a caffeine challenge to patients with panic disorder found that caffeine aggravated symptoms of anxiety and panic disorder in every study [27]. While this review provides strong evidence that caffeine can exacerbate anxiety and panic disorder, these studies were caffeine challenges and not representative of the patients' normal caffeine intake. Patterns of actual caffeine consumption among psychiatric patients have been shown to be similar to matched controls; however, maximum lifetime intake was higher among patients [28]. In addition, the prevalence of caffeine dependence and intoxication was reportedly higher in patients, who endorsed consuming more caffeine than intended, having a desire to cut down, and using caffeine despite harm more often than controls [28]. However, even among psychiatric patients, caffeine can act as a functional drug. Low to moderate daily doses of caffeine can reduce anxiety and elevate mood, and may even improve symptoms of attention-deficit hyperactivity disorder, although large-scale clinical trials have not been conducted [17]. To date, the evidence suggests that caffeine use is associated with, but does not cause, psychiatric and substance use disorders [29]. The research on caffeine use and psychiatric disorders 
raises the possibility of increased risk for CUD or caffeine intoxication due to disordered use among certain patient populations and more studies are needed on the prevalence of caffeine use among individuals with psychiatric problems.

\section{Co-Use with Other Substances}

Another potential contribution to disordered caffeine use is co-use with other substances. Caffeine may facilitate the effects of other drugs of abuse [2]. In particular, combining caffeinated energy drinks with alcoholic beverages has become a popular phenomenon because high doses of caffeine may offset the subjective intoxicating effects of alcohol; this is problematic because the objective intoxicating effects of alcohol are not affected [30]. Furthermore, the co-use of caffeine and sugary soft drinks may cause cross-sensitization, especially among children, and this could lead to poor dietary habits across the lifespan [31••]. Too many caffeinated soft drinks in one's diet could increase the risk of obesity [32] and dental caries [33] in children and adolescents.

\section{Conclusion}

In conclusion, future research on CUD must demonstrate that enough people, but not too many, meet the criteria for disordered caffeine use, and that the severity and frequency of problems resulting from this use significantly interfere with their well-being and daily function. In addition, tests of the reliability and validity of CUD criteria are needed, as well as clinical treatment options and their efficacy. If the same standard of harm can be met for caffeine as for other drugs of abuse, then perhaps in another 20 years CUD will be an official diagnosis in the sixth edition of the DSM (DSM-6). Official recognition of CUD could significantly impact popular opinions towards caffeinated beverages and affect their legal regulation. After all, 20 years ago, caffeine withdrawal was not an officially recognized diagnosis in the DSM-IV [5], but now there is sufficient evidence of caffeine withdrawal to warrant inclusion in the DSM-5. Many people are now aware that chronic caffeine use can result in physical dependence and there has been pressure on manufacturers of caffeinated beverages to disclose their products' caffeine content. Some researchers have even recommended warning labels on caffeinated beverages [34].

The roundtable discussion raised an important issue: if caffeine use were proven harmful in some capacity, then a bias may develop among researchers against discussing any of its potential benefits on health, cognition, or arousal [13••]. In this event, could there also be public backlash against caffeine consumption? If so, there may be legislative pressure to limit access to caffeine, or to apply age restrictions on who can purchase and consume caffeine, in order to reduce the likelihood of caffeine-related problems among the general population. However, considering the amount of trade and commerce surrounding caffeinated beverages, caffeine use is not only a public health concern, but a major economic concern as well. It would not be surprising if coffee, tea, soft drink, and energy drink industries took an active role in dissuading official recognition of CUD in the DSM, especially if that recognition meant increased regulation of caffeinated products.

The recent research on caffeine has important considerations for health professionals and consumers. It appears that not all consumers are aware they are dependent on caffeine, or realize that their fatigue, headache, nausea, or other symptoms are related to caffeine withdrawal, instead of an illness [13••]. Several authors recommend increasing awareness among both clinicians and patients about the relationship between caffeine use, health, and psychiatric disorders [19•, 28, 34], and also recommend including caffeine use assessments during psychiatric evaluations [26]. Importantly, the last survey of caffeine use in America was published in 2005 [35] and this information needs updating. Lastly, a survey of what clinicians are recommending to their patients regarding caffeine use would be valuable information for researchers and health professionals. In addition to these recommendations, there are many more potential avenues for future caffeine research. On the other hand, since caffeine is the most widely used psychoactive drug in the world and there are upwards of 20,000 research articles on caffeine, there may be little left to learn about this substance and our relationship with it.

Acknowledgments This work was supported by National Institutes of Health grants K01 DA033347 (National Institute on Drug Abuse).

\section{Compliance with Ethics Guidelines}

Conflict of Interest Merideth A. Addicott declares she has no conflict of interest.

Human and Animal Rights and Informed Consent This article does not contain any studies with human or animal subjects performed by the author.

\section{References}

Papers of particular interest, published recently, have been highlighted as:

- Of importance

-. Of major importance

1. American Psychiatric Association. Diagnostic and statistical manual of mental disorders, Fifth Edition, DSM-5. Arlington: American Psychiatric Association. 2013. 
2. Morelli M, Simola N. Methylxanthines and drug dependence: a focus on interactions with substances of abuse. In: Fredholm BB, editor. Handbook of experimental pharmacology. Berlin Heidelberg: Springer-Verlag; 2011. p. 483-507.

3. Nehlig A. Are we dependent upon coffee and caffeine? A review on human and animal data. Neurosci Biobehav Rev. 1999;23:563-76.

4. Satel S. Is caffeine addictive? A review of the literature. Am J Drug Alcohol Abuse. 2006;32:493-502.

5. American Psychiatric Association. Diagnostic and statistical manual of mental disorders, Fourth Edition, DSM-IV. Washington, D.C.: American Psychiatric Association. 1994.

6. Griffiths RR, Woodson PP. Caffeine physical-dependence - a review of human and laboratory-animal studies. Psychopharmacology. 1988;94:437-51.

7. Juliano LM, Griffiths RR. A critical review of caffeine withdrawal: empirical validation of symptoms and signs, incidence, severity, and associated features. Psychopharmacology. 2004;176:1-29.

8. Strain EC, Mumford GK, Silverman K, Griffiths RR. Caffeine dependence syndrome - evidence from case-histories and experimental evaluations. JAMA. 1994;272:1043-8.

9. Juliano LM, Evatt DP, Richards BD, Griffiths RR. Characterization of individuals seeking treatment for caffeine dependence. Psychol Addict Behav. 2012;26:948-54.

10. Striley CW, Griffiths RR, Cottler LB. Evaluating dependence criteria for caffeine. J Caffeine Res. 2011;1:219-25.

11. Hughes JR, Oliveto AH, Liguori A, Carpenter J, Howard T. Endorsement of DSM-IV dependence criteria among caffeine users. Drug Alcohol Depend. 1998;52:99-107.

12. Association AP. Substance-related and addictive disorders. DSM-5 Development Fact Sheets 2013. http://www.dsm5.org/Documents/ Substance $\% 20$ Use $\% 20$ Disorder $\% 20$ Fact $\% 20$ Sheet.pdf. Accessed 2 April 2014.

13.• Striley CW, Hughes JR, Griffiths RR, Juliano LM, Budney AJ. A critical examination of the caffeine provisions in the Diagnostic and Statistical Manual, 5th Edition (DSM-5). J Caffeine Res. 2013;3: 101-7. Striley moderated a roundtable discussion examining the caffeine provisions in the DSM-5. Participants were Drs. Hughes, Griffiths, Juliano, and Budney. The discussants provide an overview of the recent changes to the DSM and address the issues and concerns raised by the psychiatric community regarding those changes. Some of these concerns were that a diagnosis of caffeine withdrawal or CUD could trivialize other drug use disorders, and that disordered caffeine use could be over-diagnosed since caffeine is so widely used. The discussants emphasize that more conservative criteria are used to define caffeine withdrawal and CUD than other substance use diagnoses-to help prevent overdiagnosis - and the DSM substance use disorder diagnoses are intended for individuals who experience significant functional impairment as a consequence of their use.

14. Glade MJ. Caffeine-not just a stimulant. Nutrition. 2010;26:932-8.

15. Budney AJ, Brown PC, Griffiths RR, Hughes JR, Juliano LM. Caffeine withdrawal and dependence: a convenience survey among addiction professionals. J Caffeine Res. 2013;3:67-71.

16.• Meredith SE, Juliano LM, Hughes JR, Griffiths RR. Caffeine use disorder: a comprehensive review and research agenda. J Caffeine Res. 2013;3:114-30. Meredith et al. present a thorough review of the evidence in support of caffeine dependence and describe an agenda for future research on clinical, epidemiological, and genetic investigations of caffeine dependence and CUD. Of note, evidence is described in support of the three primary criteria for CUD, and three case reports of individuals seeking treatment for caffeine dependence are presented. The authors recommend more research on the prevalence of CUD in the general population and the severity of functional impairment associated with the disorder, as well as efficacious treatment options for affected individuals.

17. Lara DR. Caffeine, mental health, and psychiatric disorders. J Alzheimers Dis. 2010;20:S239-48.

18. McCarthy DM, Mycyk MB, DesLauriers CA. Hospitalization for caffeine abuse is associated with abuse of other pharmaceutical products. Am J Emerg Med. 2008;26:799-802. McCarthy et al. performed a retrospective review of all caffeinerelated reports to the Illinois Poison Center between 2002 and 2004. Instances of caffeine abuse were included for analysis. The authors reported that caffeine was most often abused in the form of a non-dietary medication, followed by caffeine-enhanced beverages, then dietary supplements. Forty-two percent of cases were managed in an emergency department and $13 \%$ of patients were hospitalized. The need for hospitalization was associated with concomitant abuse of other pharmaceuticals.

19. Ogawa N, Ueki H. Clinical importance of caffeine dependence and abuse. Psychiatry Clin Neurosci. 2007;61:263-8. The authors present two case reports of individuals whose caffeine use escalated to a point when they needed medical intervention for symptoms of caffeine intoxication. These case reports illustrate how caffeine use can become a clinically relevant disorder in some individuals. The authors also review evidence that caffeine abuse/dependence can manifest itself similar to other drugs of abuse. The authors recommend improved labeling of caffeinated products for consumer awareness.

20. Nawrot P, Jordan S, Eastwood J, et al. Effects of caffeine on human health. Food Addit Contam. 2003;20:1-30.

21. Palatini P, Dorigatti F, Santonastaso M, et al. Association between coffee consumption and risk of hypertension. Ann Med. 2007;39: $545-53$.

22. Klag MJ, Wang NY, Meoni LA, et al. Coffee intake and risk of hypertension - The Johns Hopkins precursors study. Arch Intern Med. 2002;162:657-62.

23. Mesas AE, Leon-Munoz LM, Rodriguez-Artalejo F, Lopez-Garcia E. The effect of coffee on blood pressure and cardiovascular disease in hypertensive individuals: a systematic review and meta-analysis. Am J Clin Nutr. 2011;94:1113-26.

24. Zhang ZZ, Hu G, Caballero B, Appel L, Chen LW. Habitual coffee consumption and risk of hypertension: a systematic review and meta-analysis of prospective observational studies. Am J Clin Nutr. 2011;93:1212-9.

25. Griffiths RR, Evans SM, Heishman SJ, et al. Low-dose caffeine physical-dependence in humans. J Pharmacol Exp Ther. 1990;255: 1123-32.

26. Winston AP, Hardwick E, Jaberi N. Neuropsychiatric effects of caffeine. Adv Psychiatr Treat. 2005;11:432-9.

27. Vilarim MM, Araujo DMR, Nardi AE. Caffeine challenge test and panic disorder: a systematic literature review. Expert Rev Neurother. 2011;11:1185-95.

28. Ciapparelli A, Paggini R, Carmassi C, et al. Patterns of caffeine consumption in psychiatric patients. An Italian study. Eur Psychiatry. 2010;25:230-5.

29. Kendler KS, Myers J, Gardner CO. Caffeine intake, toxicity and dependence and lifetime risk for psychiatric and substance use disorders: an epidemiologic and co-twin control analysis. Psychol Med. 2006;36:1717-25.

30. Ferreira SE, de Mello MT, Pompeia S, de Souza-Formigoni MLO. Effects of energy drink ingestion on alcohol intoxication. Alcohol Clin Exp Res. 2006;30:598-605.

31.• Temple JL. Caffeine use in children: what we know, what we have left to learn, and why we should worry. Neurosci Biobehav Rev. 2009;33:793-806. Review of caffeine literature, including sources of caffeine and consumption patterns; physiological mechanisms; evidence for addiction, tolerance, and sensitization; and caffeine 
conditioning and reinforcement value. A special emphasis is given to caffeine use among children and adolescents. In particular, cross-sensitization between caffeine and sugary beverages could set up poor dietary habits across the lifespan. Also, children and adolescents may experience pressure in the classroom and from coaches to drink caffeine for its putative performance-enhancing effects. The author concludes that research is lacking on caffeine use in children and adolescents, but the long-term effects of caffeine exposure during a critical period of development may be a cause for concern.
32. Ludwig DS, Peterson KE, Gortmaker SL. Relation between consumption of sugar-sweetened drinks and childhood obesity: a prospective, observational analysis. Lancet. 2001;357:505-8.

33. Majewski RF. Dental caries in adolescents associated with caffeinated carbonated beverages. Pediatr Dent. 2001;23:198-203.

34. Reissig CJ, Strain EC, Griffiths RR. Caffeinated energy drinks-a growing problem. Drug Alcohol Depend. 2009;99:1-10.

35. Frary CD, Johnson RK, Wang MQ. Food sources and intakes of caffeine in the diets of persons in the United States. J Am Diet Assoc. 2005;105:110-3. 